\title{
A Robust Filtering Algorithm of LiDAR Data for DTM Extraction
}

\author{
MING Yang ${ }^{1, a}$, CHEN Chujiang ${ }^{1, b}$
}

\author{
${ }^{1}$ Geospatial Information and Digital Transportation Research Institute, CCCC Second Highway \\ Consultants Co., Ltd, Wuhan, China

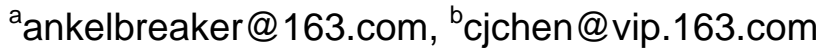

Keywords: LiDAR; ground filtering; digital terrain model

\begin{abstract}
For DTM extraction of road linear project from LiDAR data, this paper proposes a robust ground filtering algorithm. Firstly, a pyramidal hierarchical structure of ground candidate points is formed. Secondly, in each level, the Thin Plate Spline (TPS) surface interpolation is iteratively used to produces smooth surfaces, and based on the point's residuals from the surface the ground candidate points are inspected. The experiments show that this method correctly determines DTM even in those cases of heavy vegetation, and achieve a better result for the road.
\end{abstract}

\section{Introduction}

Light detection and ranging (LiDAR) technology has revolutionized conventional methods for spatial data acquisition. Mounted on aircraft or mobile, LiDAR system is capable of gathering Earth's terrain data rapidly with high accuracy and great density. It is also an active remote sensing system, and is able to penetrate through vegetation to record the terrain beneath it. In the linear project, such as road construction, the LiDAR technology has been gradually widely applied.

One main step in the processing of LiDAR data is the separation of ground and non-ground points, and then to generate the Digital Terrain Model (DTM). Various methods have been proposed so far $^{[1-2]}$. Morphological filtering algorithm has been proposed ${ }^{[3-4]}$, in which the structure element is used to describe admissible height differences in certain horizontal distance. Vosselman proposed an optimal structure element to minimize type I and type II errors ${ }^{[3]}$, and Sithole proposed a terrain shape depended structure element, which allowed higher admissible height differences in steeper areas $^{[4]}$. But the common weakness of these methods is additional knowledge about the extent of the contained objects and suitable threshold values are required.

Axelsson generated DEM from laser scanner data using adaptive TIN models ${ }^{[5]}$, the lowest points in large grid cells are used as the initial ground points, which are triangulated to form the approximate surface, that is so called reference surface. For each unclassified point, the offsets to the reference surface are inspected, when the offsets are below the predefined threshold values, it is classified as ground point. Iteratively processed, the reference surface become much finer and the ground points are progressively densified. Based on the similar idea, Sohn realized the surface reconstruction by the use of tetrahedron model with the MDL criterion ${ }^{[6]}$.

Another important kind of algorithms is based on surface interpolation ${ }^{[7-9]}$. Kraus and Pfeifer use linear prediction based methods to interpolate surface for determining a buffer-zone within which ground points are accepted. But since only a rough surface approximation is obtained by linear interpolation, it tends to misclassify minute objects and do not preserve terrain details.

In this paper, a robust ground filtering algorithm is proposed, which integrates the Thin Plate Spline surface interpolation and hierarchic strategy to iteratively densify the ground points. Experiments have been carried out, and good results are obtained.

\section{Proposed algorithm}

Point Cloud Pyramid. To copy with the problem of huge data volume and gross errors in larger areas, the point cloud pyramid is formed before the filtering process. The input point cloud $P=\left\{p_{\mathrm{i}}\right\}$ is firstly arranged into a grid $G$. The resolution of $G$ is related to the desired resolution of the resulting DTM. Each grid cell includes an array of contained points. Since the ground is considered 
to be a smooth continuous surface with nothing beneath it, the lowest point in each cell is selected as the ground point candidate.

The hierarchy is formed in a bottom-up fashion, where successive levels are produced by down-sampling the previous levels by a factor of two. That is, a particular cell at a given level is derived from the $2 \times 2$ neighboring cells at the lower level, and only the lowest points within these cells is selected. The construction is finished when the size of the cell is larger than the expected sizes of the contained objects.

Thin Plate Spline Surface Interpolation. Thin plate Spline interpolation produces smooth, oscillation-free surfaces. For a given set of irregularly distributed non-coplanar control points, TPS interpolation finds a smooth surface $S(x, y)$ that interpolates all the given control-points at minimized bending energy $J_{\mathrm{s}}$ given by Zandifar ${ }^{[10]}$.

$$
J_{s}=\iint_{R^{2}}\left[\left(\frac{\partial^{2} S}{\partial x^{2}}\right)^{2}+2\left(\frac{\partial^{2} S}{\partial x \partial y}\right)^{2}+\left(\frac{\partial^{2} S}{\partial y^{2}}\right)^{2}\right] d x d y
$$

The surface is expressed as

$$
s(x, y)=a_{0}+a_{1} x+a_{2} y+\sum_{i=1}^{N} w_{i} R\left(\left\|\left(x_{i}, y_{i}\right)-(x, y)\right\|\right)
$$

Where the kernel function $R$ is defined as $R(r)=r^{2} \log r$, and $w_{i}$ are weights related to the control-points. In order for $S$ to have square integrable second derivatives, the conditions $\sum_{i=1}^{N} w_{i}=\sum_{i=1}^{N} w_{i} x_{i}=\sum_{i=1}^{N} w_{i} y_{i}=0$ should be met. $S$ is obtained by estimating column vector $a$ and $w$ by the following linear system:

$$
\left[\begin{array}{cc}
K & P \\
P^{T} & 0
\end{array}\right]\left[\begin{array}{l}
w \\
a
\end{array}\right]=\left[\begin{array}{l}
T \\
0
\end{array}\right]
$$

Where $K$ is an $N \times N$ matrix defined by $K_{i j}=R\left(\left\|\left(x_{i}, y_{i}\right)-(x, y)\right\|, P\right.$ is an $N \times 3$ matrix, where the $i$ th row is formulate as $\left(1, x_{i}, y_{i}\right)$, and $T$ is a tensor of the control points.

Hierarchic Robust Ground Filtering. To alleviate the calculation burden, merely the ground point candidates are used to generate the DTM and the LiDAR points are only filtered at the finest level.

The hierarchic interpolation proceeds like the following: 1) Starting at the coarsest level, the TPS interpolation method is iteratively used to generate the DTM in this level; 2) calculate the offsets to the DTM of the ground point candidates from the next finer level, and accept points within a certain tolerance band; 3) the TPS interpolation is iteratively used to derive the DTM in current level; 4) the step 2 and step 3 are repeated until generating the DTM in the finest level; 5) the original points are compared the generated DTM and the ground points are finally derived.

\section{Experiment Results and Analysis}

To assess the performance of the algorithm proposed in this paper, two datasets acquired from the airborne LiDAR are tested. To obtain the road spatial information of high accuracy and great density, the LiDAR data is collected by a helicopter, with a relatively low fly height, and the point spacing is just $0.15 \mathrm{~m}$, that is there are about 36 points per square meter. Fig. 1 depicts the non-filter DSM for both datasets, colored by point intensity. As can be seen from the figure, for two datasets, the both sizes of the road are heavily covered by the vegetation. 


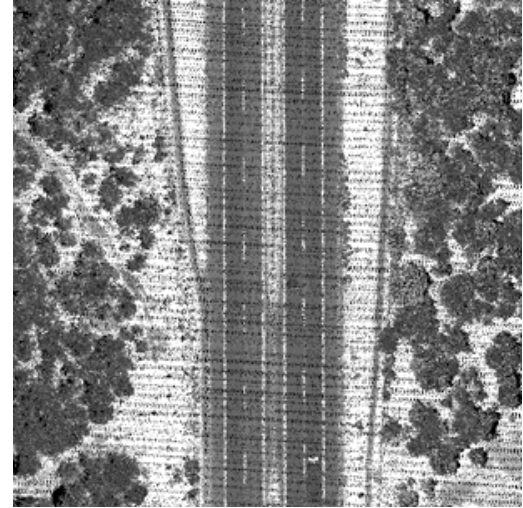

(a) dataset one

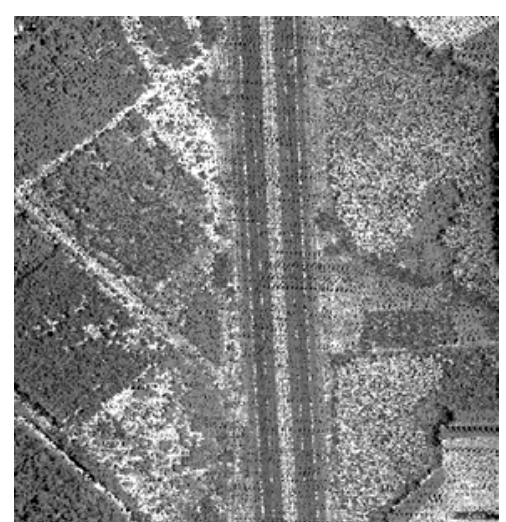

(b) dataset two

Fig. 1 Non filter DSM colored by the point intensity

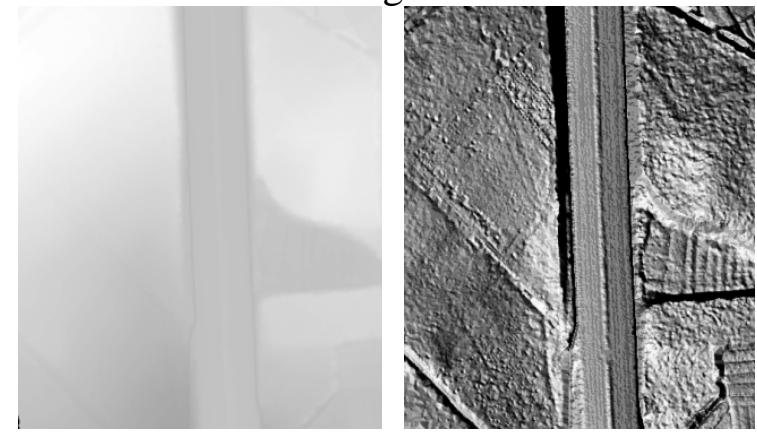

(a) dataset one (b) dataset two

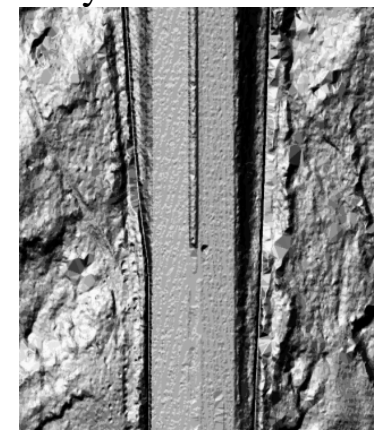

Fig. 2 Filtered DTM extracted by the method

For dataset one and two, the total number of points is 1,399,770 and 1,755,729 respectively, after applying the algorithm, about 314,718 and 312,497 points are classified to the ground points. When compared to the method proposed by the Axelsson, which only classified the lowest point as ground point, the number of ground point is 168,194 and 196,133 for dataset one and two respectively. That is because the points in the buffer-zone near the TPS interpolation surface will all be accepted as ground points, which enable us to extract more road points. Fig. 2 shows the extracted DTM, it can be seen that the overall terrain is smooth and continuous, road surfaces are well reconstructed and the terrain feature are also perserved with great detail.

\section{Conclusions}

This paper presents a robust ground filtering algorithm for DTM extraction. The hierarchical Thin Plate Spline (TPS) interpolation is used to generate the DTM in each level. To alleviate the calculation burden, merely the ground point candidates are used to generate the DTM and the LiDAR points only filtered at the finest level. The experiments have demonstrated that the method can obtain satisfactory result, can derive good spatial terrain information for the road construction project.

[1] G. Sithole, G. Vosselman, Experimental comparison of filter algorithms for bare-Earth extraction from airborne laser scanning point clouds, ISPRS Journal of Photogrammetry and Remote Sensing. 59 (2004) 85-101.

[2] Q. Chen, Assessment of terrain elevation derived from satellite laser altimetry over mountainous forest areas using airborne lidar data, ISPRS Journal of Photogrammetry and Remote Sensing, 65 (2010) 111-122.

[3] G. Vosselman, Slope based filtering of laser altimetry data, International Archives of Photogrammetry and Remote Sensing, 33 (2000) 935-942.

[4] G. Sithole, Filtering of laser altimetry data using a slope adaptive filter, International Archives 
of Photogrammetry and Remote Sensing, 34 (2001) 203-210.

[5] P. Axelsson, DEM generation from laser scanner data using adaptive TIN models, International Archives of Photogrammetry and Remote Sensing, 33 (2000) 110-117.

[6] G. Sohn, I. Dowman, Terrain surface reconstruction by the use of tetrahedron model with MDL criterion, International Archives of Photogrammetry and Remote Sensing, 34 (2002) 336-344.

[7] K. Kraus, N. Pfeifer, Determination of terrain models in wooded areas with airborne laser scanner data, ISPRS Journal of Photogrammetry and Remote Sensing, 53 (1998) 193-203.

[8] N. Pfeifer, T. Reiter, C. Bries, W. Rieger, Interpolation of high quality ground models from laser scanner data in forested areas, International Archives of Photogrammetry and Remote Sensing, 32 (1999) 31-36.

[8] A. Kobler, N. Pfeifer, P. Ogrinc, et al., Repetive interpolation: a robust algorithm for DTM generation from aerial laser scanner data in forested terrain, Remote Sensing of Environment, 108 (2007) 9-23.

[10] A. Zandifar, S. Lim, R. Duraiswami, N. Gumerov, L. Davis, Multi-level fast multipole method for thin plate spline evaluation, Proceedings of International Conference on Image Preocessing, 3 (2004) 1683-1686. 